\title{
Dynamic Response of Plasma Detachment Caused by Molecular Activated Recombination to Transient Particle Flux in GAMMA 10/PDX ${ }^{*}$
}

\author{
Akihiro TERAKADO, Mizuki SAKAMOTO, Naomichi EZUMI, Kunpei NOJIRI, \\ Tomohiro MIKAMI, Yosuke KINOSHITA, Satoshi TOGO, \\ Takaaki IIJIMA and Yousuke NAKASHIMA \\ Plasma Research Center, University of Tsukuba, Tsukuba 305-8577, Japan
}

(Received 30 September 2018 / Accepted 9 December 2018)

\begin{abstract}
The dynamic response to a particle flux in a divertor simulation plasma has been studied using electron cyclotron heating (ECH) in GAMMA 10/PDX. Hydrogen gas was supplied to the divertor simulation experimental module (D-module), and ECH was applied to the main plasma. The electron temperature $\left(T_{\mathrm{e}}\right)$ decreased, and a rollover of electron density $\left(n_{\mathrm{e}}\right)$ was observed upon an additional supply of hydrogen gas with and without $\mathrm{ECH}$, which indicates that the plasma was detached. Moreover, the intensity of $\mathrm{H}_{\alpha}\left(I_{\mathrm{H} \alpha}\right)$ increased even though $n_{\mathrm{e}}$ decreased with an increasing gas supply, indicating that the plasma detached due to molecular activated recombination (MAR). In the case of additional gas supply, $n_{\mathrm{e}}$ increased, but $T_{\mathrm{e}}$ was not affected by ECH. The ionization front moved to the upstream side, and the particle flux flowing into the D-module increased with ECH. The ratio of $I_{\mathrm{H} \alpha}$ to the intensity of $\mathrm{H}_{\beta}\left(I_{\mathrm{H} \beta}\right)$ near the corner of the $\mathrm{V}$-shaped target increased with $\mathrm{ECH}$, indicating that the MAR was enhanced near the corner. The intensity of the Fulcher- $\alpha$ Q1-branch increased near the corner of the $\mathrm{V}$-shaped target with $\mathrm{ECH}$, indicating that the density of hydrogen molecules was reduced by hydrogen recycling.

(C) 2019 The Japan Society of Plasma Science and Nuclear Fusion Research
\end{abstract}

Keywords: GAMMA 10/PDX, divertor simulation experiment, plasma detachment, molecular activated recombination, dynamic response

DOI: $10.1585 /$ pfr. 14.2402109

\section{Introduction}

Divertor detachment is the most promising solution for reducing the heat load on a divertor plate. Volumetric recombination, such as electron-ion recombination (EIR) and molecular activated recombination (MAR), plays an important role in plasma detachment [1]. In the H-mode discharge of tokamaks, the edge-localized mode (ELM) releases a large amount of energy from the core plasma to the divertor region, which breaks up the plasma detachment and significantly affects the divertor plate. Understanding the effects of upstream heat and particle flux on plasma detachment is an important research aim. The response of plasma detachment caused by EIR and MAR to heat and particle flux, such as the ELM, has been investigated [1-4]. However, little has been reported on the dynamic response of the spatial distribution of plasma detachment due to MAR.

In GAMMA 10/PDX, divertor plasma phenomena has been studied using an end-loss plasma as a divertor simulation plasma [5]. It was found that the divertor simulation plasma is detached by MAR related to triatomic molecular ions [6]. In this study, the dynamic response of the plasma

author'se-mail: terakado_akihiro@prc.tsukuba.ac.jp

*) This article is based on the presentation at the 12th International Conference on Open Magnetic Systems for Plasma Confinement (OS2018). detachment caused by MAR to transient heat and particle flux has been examined utilizing electron cyclotron heating $(\mathrm{ECH})$. In addition, changes in the spatial distribution of the detached plasma due to transient heat and particle flux are discussed.

\section{Experimental Setup}

Figure 1 shows a schematic view of the tandem mirror GAMMA 10/PDX, consisting of a central cell, anchor cells, plug/barrier cells, and end regions [7]. The Dmodule is installed in the west end region $[8,9]$ and consists of a rectangular box with dimensions of $480 \mathrm{~mm} \times$ $500 \mathrm{~mm} \times 700 \mathrm{~mm}$. A quartz window is attached on the side of the D-module to measure the emission of the diver-

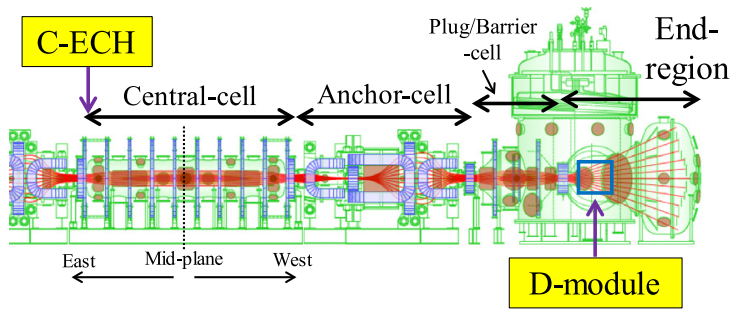

Fig. 1 Schematic view of GAMMA 10/PDX. 
tor simulation plasma. A V-shaped target is installed inside the D-module, and the plasma-facing material of the $\mathrm{V}$ shaped target is tungsten. The V-shaped target is exposed to the end-loss plasma that flows into the D-module. Additional gas can be supplied toward the V-shaped target from a position near the plasma inlet.

Figure 2 (a) shows the top view of the west end region and the line of sight of a high-speed camera and spectroscopy equipment. Langmuir probes are installed on the surface of the upper target plate and corner of the $\mathrm{V}$-shaped target and are defined as A, B, and C from the corner of the $\mathrm{V}$-shaped target toward the upstream side, as shown in Fig. 2 (b). The electron temperature $\left(T_{\mathrm{e}}\right)$ and electron density $\left(n_{\mathrm{e}}\right)$ are measured by the Langmuir probes. Hydrogen Balmer line intensities are measured with a low-dispersion spectrometer (USB2000+, Ocean Optics), and the Q1branch intensity of the Fulcher- $\alpha$ band is measured with a high-dispersion spectrometer (SR500i, ANDOR). The measurement positions of the SR500i are defined as No. 1 and No. 2 from the corner of the V-shaped target directed upstream. The relative sensitivity of the spectrometers was calibrated using a standard lamp. Two-dimensional images of the hydrogen Balmer-alpha intensity $\left(I_{\mathrm{H} \alpha}\right)$ and Balmerbeta intensity $\left(I_{\mathrm{H} \beta}\right)$ in front of the V-shaped target are measured using a high-speed camera with an interference filter placed in front of the lens. The center wavelengths of the transmittance profile of the interference filter for $I_{\mathrm{H} \alpha}$ and $I_{\mathrm{H} \beta}$ are $656 \mathrm{~nm}$ and $486 \mathrm{~nm}$, respectively. The full width at half maximum of both filters is $10 \mathrm{~nm}$.

(a)
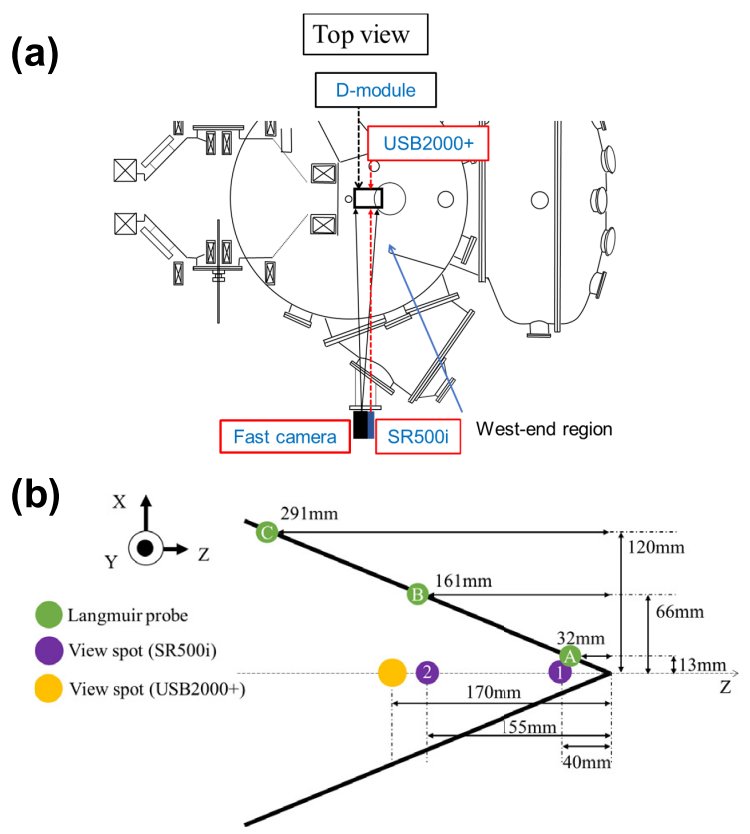

Fig. 2 (a) Top view of the west end region and line of sight of the spectrometers (USB2000+ and SR500i); and (b) measurement positions of Langmuir probes and USB2000+ and SR500i.

\section{Results and Discussion}

The main plasma was produced and maintained from $t=50 \mathrm{~ms}$ to $t=250 \mathrm{~ms}$ by ion cyclotron resonance frequency (ICRF) heating at $\sim 290 \mathrm{~kW}$. Additional hydrogen gas was supplied to the D-module from $300 \mathrm{~ms}$ before the plasma production until the end of plasma discharge. The amount of additional hydrogen gas was changed shot by shot by changing the pressure in the reservoir tank (i.e., plenum pressure) at the upstream side of a piezoelectric valve. ECH was applied in the central cell in order to measure the dynamic response of the divertor simulation plasma. The ECH was applied from $160 \mathrm{~ms}$ to $185 \mathrm{~ms}$ at $70 \mathrm{~kW}$. The electron line density and diamagnetism increase during ECH, as shown in Fig. 3 (a). The electron temperature, which was measured at the central cell by Thomson scattering, increases from approximately $10 \mathrm{eV}$ to $47 \mathrm{eV}$ due to $\mathrm{ECH}$, as shown in Fig. 3 (b) for a plenum pressure of 600 mbar.

Figure 4 shows the electron line density at the plug cell, $T_{\mathrm{e}}, n_{\mathrm{e}}, I_{\mathrm{H} \alpha}$, and $I_{\mathrm{H} \beta}$ in the D-module as a function of plenum pressure with and without ECH. In both cases, the electron line density increases with increasing plenum pressure, which is caused by the ionization of hydrogen neutrals escaping from the D-module. When the plenum pressure exceeds 400 mbar, the electron line density is increased by ECH, as shown in Fig. 4 (a). The electron temperature is not affected by ECH when hydrogen gas is supplied. The value of $T_{\mathrm{e}}$ decreases to $\sim 4 \mathrm{eV}$ as the plenum pressure increases. The electron density increases with increasing plenum pressure until approximately $200 \mathrm{mbar}$ and then decreases (i.e., density rollover) both with and without ECH. The decrease in $T_{\mathrm{e}}$ and the density rollover indicate that the plasma was detached. As Fig. 4 (c) shows, the electron density with ECH is larger than that without ECH when hydrogen gas is supplied. The increase in $n_{\mathrm{e}}$ upon $\mathrm{ECH}$ at a plenum pressure of $200 \mathrm{mbar}$ is caused by

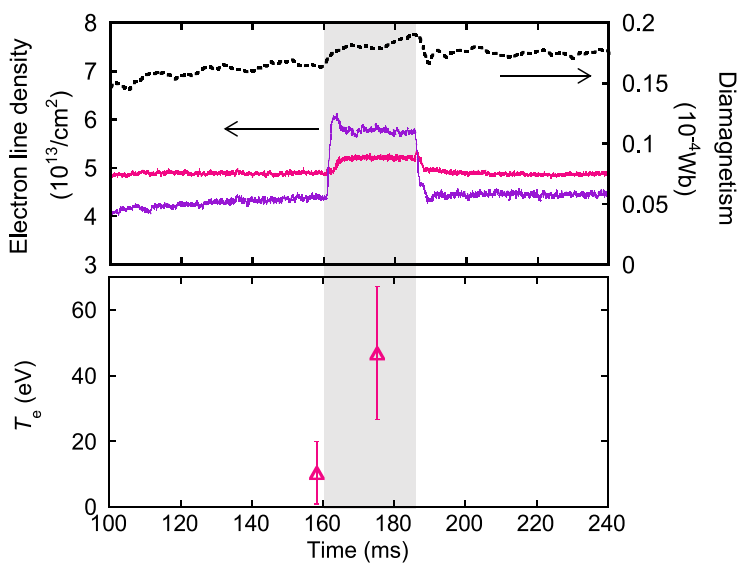

Fig. 3 Time evolution of (a) the electron line densities at the central cell and plug cell plasmas, and diamagnetism of the central cell plasma, (b) the electron temperature of the central cell plasma. 

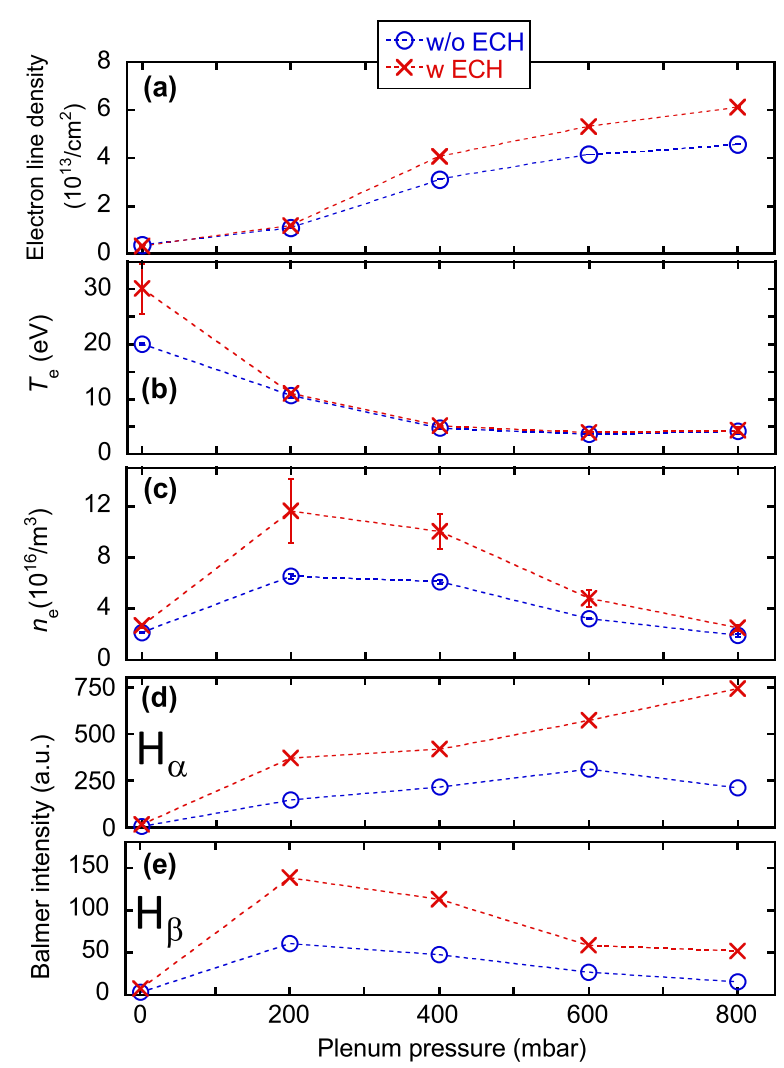

Fig. 4 (a) Electron line density at the plug cell, (b) Electron temperature, (c) electron density, (d) $\mathrm{H}_{\alpha}$ and (e) $\mathrm{H}_{\beta}$ intensities as a function of the plenum pressure. Electron temperature and electron density are measured by a Langmuir probe at the position of $\mathrm{A}$ and Balmer intensity measured by spectroscopy (USB2000+). The dashed lines are a guide to the eye.

ionization in the D-module because the line density of the plug cell does not change with ECH. In contrast, when the plenum pressure exceeds $400 \mathrm{mbar}, n_{\mathrm{e}}$ for the D-module increases due to ionization in the D-module and the increased particle flux flowing from upstream (i.e., the plugcell) to the D-module.

The dependence of $I_{\mathrm{H} \beta}$ on plenum pressure is similar to that of $n_{\mathrm{e}}$ both with and without ECH. However, $I_{\mathrm{H} \alpha}$ continues to increase with increasing plenum pressure up to 800 mbar even though $n_{\mathrm{e}}$ decreases in the absence of $\mathrm{ECH}$. In this case, $I_{\mathrm{H} \alpha}$ increases with increasing plenum pressure up to $600 \mathrm{mbar}$ and then decreases. The continuous increase in $I_{\mathrm{H} \alpha}$ is primarily caused by MAR [6]. The decrease in $I_{\mathrm{H} \alpha}$ at 800 mbar without $\mathrm{ECH}$ is attributed to a decreased vibrational temperature of the molecules [10].

Figure 5 shows the time evolution of $T_{\mathrm{e}}$ and $n_{\mathrm{e}}$ at a plenum pressure of 600 mbar. The electron temperature at probe $\mathrm{C}$ decreased upon $\mathrm{ECH}$, but $T_{\mathrm{e}}$ at probes $\mathrm{A}$ and $\mathrm{B}$ remained constant. The electron density at the three positions increased due to $\mathrm{ECH}$, and the increase in $n_{\mathrm{e}}$ was approximately 1.5-2 folds at each probe. The difference between the $n_{\mathrm{e}}$ values for probes $\mathrm{B}$ and C decreased upon
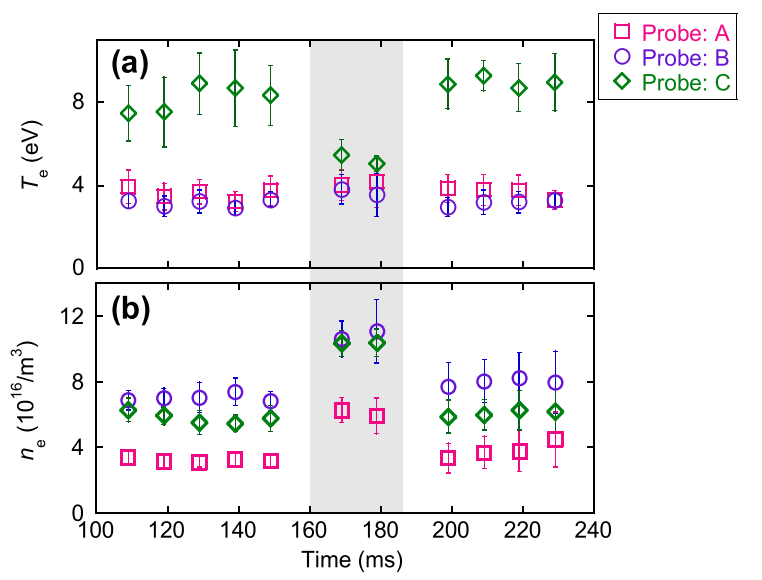

Fig. 5 Time evolution of (a) the electron temperature and (b) the electron density on the V-shaped target.
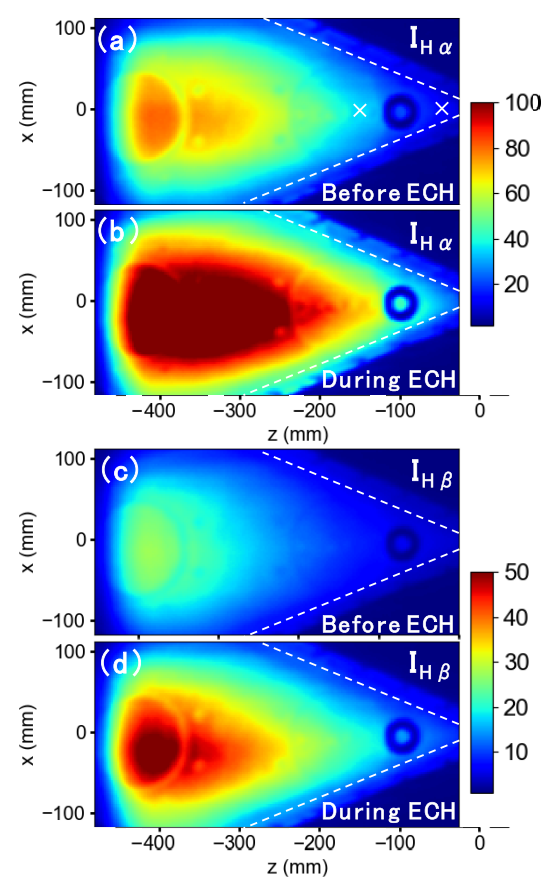

Fig. 6 Two-dimensional images of Balmer line intensities in front of the V-shaped target measured by the high-speed camera. (a) $I_{\mathrm{H} \alpha}$ before $\mathrm{ECH}$ and (b) $I_{\mathrm{H} \beta}$ during $\mathrm{ECH}$. (c) $I_{\mathrm{H} \alpha}$ before $\mathrm{ECH}$ and (d) $I_{\mathrm{H} \beta}$ during ECH. White dash lines indicate the surface of $\mathrm{V}$-shaped target.

$\mathrm{ECH}$, indicating that the spatial distribution of $n_{\mathrm{e}}$ changes. Thus, the ionization front is moved the upstream by $\mathrm{ECH}$. The decreased $T_{\mathrm{e}}$ of probe $\mathrm{C}$ is attributed to the movement of the ionization front to the upstream side. The electron temperatures at probes A and B were not affected by ECH because the plasma was already detached before $\mathrm{ECH}$.

Figure 6 shows two-dimensional images of $I_{\mathrm{H} \alpha}$ and $I_{\mathrm{H} \beta}$ with and without $\mathrm{ECH}$ for a plenum pressure of 600 mbar. The concentric structure near the corner of the $\mathrm{V}$-shaped target $(x \sim 0 \mathrm{~mm}, z \sim 80 \mathrm{~mm})$ is the lens for Thom- 

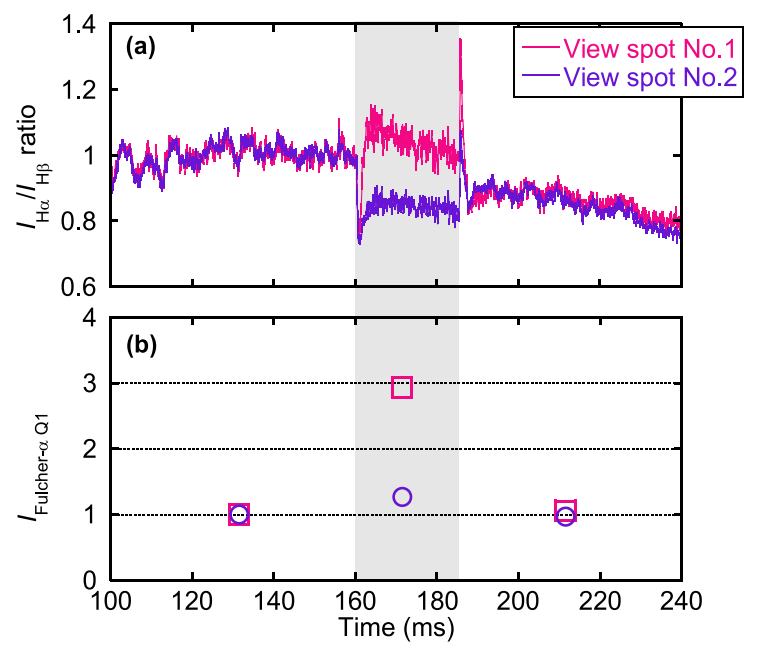

Fig. 7 Time evolution of (a) $I_{\mathrm{H} \alpha} / I_{\mathrm{H} \beta}$ and (b) $I_{\mathrm{Q} 1}$ at view spot of No. 1 and No. 2. Each value is normalized by the value of without $\mathrm{ECH}$.

son scattering measurements. The values of $I_{\mathrm{H} \alpha}$ and $I_{\mathrm{H} \beta}$ decrease from the upstream side to the corner of the $\mathrm{V}$ shaped target both with and without $\mathrm{ECH}$, indicating that the plasma is detached. The values of $I_{\mathrm{H} \alpha}$ and $I_{\mathrm{H} \beta}$ in front of the V-shaped target are increased by $\mathrm{ECH}$, which is attributed to the increase in $n_{\mathrm{e}}$.

Figure 7 (a) shows the time evolution of $I_{\mathrm{H} \alpha} / I_{\mathrm{H} \beta}$ near the corner of the V-shaped target $(x=0 \mathrm{~mm}, z=-40 \mathrm{~mm})$ and near the upstream side $(x=0 \mathrm{~mm}, z=-170 \mathrm{~mm})$. The data for $I_{\mathrm{H} \alpha} / I_{\mathrm{H} \beta}$ in Fig. 7 (a) are normalized by the values at $159 \mathrm{~ms}$ (i.e., normalized by $I_{\mathrm{H} \alpha} / I_{\mathrm{H} \beta}$ without $\mathrm{ECH}$ ). The positions near the corner of the $\mathrm{V}$-shaped target $(x=$ $0 \mathrm{~mm}, z=-40 \mathrm{~mm})$ and the upstream side $(x=0 \mathrm{~mm}$, $z=-170 \mathrm{~mm}$ ) are hereinafter defined as measurement positions No. 1 and No. 2. The positions of measurement positions No. 1 and No. 2 are the same as those of the SR500i spectroscope shown in Fig. 2 (b). The ratio of $I_{\mathrm{H} \alpha} / I_{\mathrm{H} \beta}$ can be used to monitor the occurrence of MAR in the detached plasma [11]. As Fig. 7 (a) shows, the ratio of $I_{\mathrm{H} \alpha} / I_{\mathrm{H} \beta}$ at measurement position No. 1 decreased immediately after $\mathrm{ECH}$ and then increased. In contrast, the ratio at measurement position No. 2 was reduced by ECH. Thus, the occurrence of MAR increased at measurement position No. 1 but decreased at No. 2. The rapid decrease and increase in $I_{\mathrm{H} \alpha} / I_{\mathrm{H} \beta}$ immediately after the ECH starts and stops may be caused by a difference in the production processes of excited atoms with $n=3$ and $n=4$, which appear to be produced by MAR processes.

Figure 7 (b) shows the Fulcher- $\alpha$ Q1-branch intensity $\left(I_{\mathrm{Q} 1}\right)$ normalized by the value at $132 \mathrm{~ms}$ (without $\mathrm{ECH}$ ). The value of $I_{\mathrm{Q} 1}$ at No. 1 increases 3-fold due to $\mathrm{ECH}$ whereas the increase in $I_{\mathrm{Q} 1}$ at No. 2 is approximately 1.3-fold. The intensities of the Q1-branch are proportional to the electron density, the molecular density, and the rate coefficient of electron-impact excitation given as

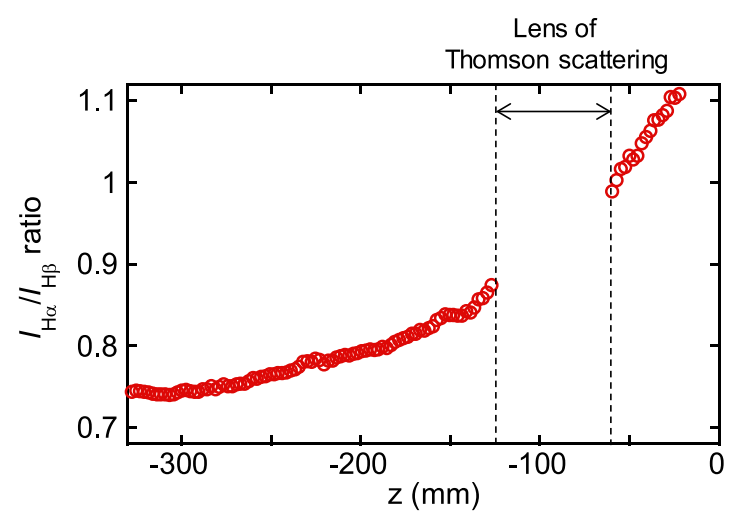

Fig. 8 Spatial distribution of $I_{\mathrm{H} \alpha} / I_{\mathrm{H} \beta}$ ratio on the $z$-axis at $t=$ $164 \mathrm{~ms}$. It is normalized by the value of without $\mathrm{ECH}$ $(t=159 \mathrm{~ms})$.

$\mathrm{H}_{2}\left(\mathrm{X}^{1} \Sigma_{\mathrm{g}}^{+}\right) \rightarrow \mathrm{H}_{2}\left(\mathrm{~d}^{3} \Pi_{\mathrm{u}}^{-}\right)$. The rate coefficient depends on the vibrational level at the electronic ground state $\left(\mathrm{X}^{1} \Sigma_{\mathrm{g}}^{+}\right)$ as well as $T_{\mathrm{e}}$. As Fig. 5 (a) shows, the electron temperature did not change due to ECH. The increase in $n_{\mathrm{e}}$ upon ECH is approximately 1.5-fold, as shown in Fig. 5 (b). The vibrational level of the hydrogen molecules is not considered to depend on the change in particle flux due to ECH. Therefore, the significant increase in $I_{\mathrm{Q} 1}$ at No. 1 upon ECH is attributed to the increased density of hydrogen molecules, resulting from the increased hydrogen recycling flux near the corner of the V-shaped target due to the increased flux. The mean free path for MAR of $\mathrm{H}_{2}$ is rather short (i.e., less than several millimeters) when $T_{\mathrm{e}}$ is $\sim 3 \mathrm{eV}$ and $n_{\mathrm{e}}$ is $\sim 10^{16} / \mathrm{m}^{3}$. Hence, the increased MAR caused by ECH was localized in the region close to the target due to an increased recycling flux.

Figure 8 shows the spatial distribution of $I_{\mathrm{H} \alpha} / I_{\mathrm{H} \beta}$ along the $z$-axis. In Fig. 8 , the values of $I_{\mathrm{H} \alpha} / I_{\mathrm{H} \beta}$ are normalized by the values at $159 \mathrm{~ms}$ (i.e., without $\mathrm{ECH}$ ), as in Fig. 7 (a). The value of $I_{\mathrm{H} \alpha} / I_{\mathrm{H} \beta}$ increases from the upstream side to the corner of the $\mathrm{V}$-shaped target in the case of ECH. In the region near the corner (i.e., $-57 \mathrm{~mm}<$ $z<-10 \mathrm{~mm}), I_{\mathrm{H} \alpha} / I_{\mathrm{H} \beta}$ is larger than 1, indicating that the occurrence of MAR increased near the corner $(-57 \mathrm{~mm}<$ $z<-10 \mathrm{~mm}$ ) and decreased toward the upstream side upon $\mathrm{ECH}$.

\section{Summary}

The dynamic response of the plasma detachment caused by MAR in a divertor simulation plasma was investigated by utilizing ECH. In this experiment, hydrogen gas was supplied to the D-module to produce detached plasma due to MAR, and ECH was applied to the main plasma. When an additional gas supply was incorporated, $n_{\mathrm{e}}$ increased, but $T_{\mathrm{e}}$ was not affected by ECH. The ionization front moved to the upstream side due to ECH. The ratio of $I_{\mathrm{H} \alpha} / I_{\mathrm{H} \beta}$ near the corner of the $\mathrm{V}$-shaped target $(-57 \mathrm{~mm}<z<-10 \mathrm{~mm})$ increased upon ECH, thus, indi- 
cating that MAR is enhanced near the corner. The increase in $I_{\mathrm{Q} 1}$ near the corner of the $\mathrm{V}$-shaped target due to $\mathrm{ECH}$ indicates an increased density of hydrogen molecules resulting from hydrogen recycling.

\section{Acknowledgments}

This study was supported by the bidirectional collaboration research program of the University of Tsukuba and the National Institute for Fusion Science (NIFS16KUGM119).

[1] S. Takamura et al., Plasma Sources Sci. Technol. 11, A42
(2002).

[2] A. Loarte et al., Nucl. Fusion 38, 331 (1998).

[3] N. Ohno et al., Nucl. Fusion 41, 1055 (2001).

[4] T. Iijima et al., Plasma Fusion Res. 9, 2405010 (2014).

[5] Y. Nakashima et al., Nucl. Fusion 57, 116033 (2017).

[6] M. Sakamoto et al., Nucl. Mater. Energy 12, 1004 (2017).

[7] M. Inutake et al., Phys. Rev. Lett. 55, 939 (1985).

[8] Y. Nakashima et al., Trans. Fusion Sci. Technol. 63, No.1T, 100 (2013).

[9] M. Sakamoto et al., Trans. Fusion Sci. Technol. 63, No.1T, 188 (2013).

[10] A. Terakado et al., Nucl. Mater. Energy. In press (2018). https://doi.org/10.1016/j.nme.2019.100679

[11] A. Terakado et al., AIP Conf. Proc. 1771, 20002 (2016). 